\title{
Effects of aerobic exercise training and cranial electrotherapy stimulation on the stress-related hormone, the neurotrophic factor, and mood states in obese middle-aged women: a pilot clinical trial
}

\author{
Su-Youn Cho, ${ }^{1}$ Wi-Young So, ${ }^{2}$ Hee-Tae Roh ${ }^{3}$
}

Original article

\section{ABSTRACT}

Introduction

Obesity, a global pandemic, has been reported as a potential cause of various diseases, including high blood pressure, type- 2 diabetes, and cardiovascular disease.

\section{Objective}

The purpose of the present research was to investigate the effects of eight weeks of regular aerobic exercise and cranial electrotherapy stimulation (CES) on the stress-related hormone, the neurotrophic factor, and mood states in obese women.

\section{Method}

The subjects for this study were 36 obese middle-aged Korean women. Subjects were randomly divided into a control group (CON, $n=12)$, an aerobic exercise group ( $E X, n=12$ ), and an aerobic exercise and CES group ( $E X+C E S, n=12$ ). CES was conducted with a micro current cranial electrotherapy stimulator for 20 minutes at $100 \mu \mathrm{A}$ and $0.5 \mathrm{~Hz}$. Aerobic exercise consisted of treadmill running for 40 minutes at $70 \%$ of the subjects' heart rate reserve (HRR). Mood state was measured by the profile of mood states (POMS) questionnaire, and blood collection was performed to examine levels of stress-related hormones (cortisol and adrenocorticotropic hormone [ACTH]) and neurotrophic factors (brain-derived neurotrophic factor [BDNF] and nerve growth factor [NGF]) before and after each intervention.

\section{Results}

Plasma cortisol levels, as well as scores for Tension-Anxiety, Depression-Dejection, and Fatigue-Inertia were significantly decreased after intervention when compared with pre-intervention measurements in the EX and EX+CES groups $(p<.05)$. Serum BDNF levels, serum NGF levels, and Vigor-Activity scores were significantly increased after intervention when compared with pre-intervention measurements in the EX and EX+CES groups $(p<.05)$. However, there was no significant difference in these findings between the EX and EX+CES groups $(p>$.05).

\section{Discussion and conclusion}

These results suggest that aerobic exercise training could improve the mood state of obese middle-aged women through a decrease in serum cortisol and an increase in serum BDNF and NGF.

Key words: Aerobic exercise, cranial electrotherapy stimulation, mood, obesity.

\section{RESUMEN}

\section{Introducción}

La obesidad, una pandemia mundial, se ha reportado como una posible causa de diversas enfermedades, incluyendo presión arterial alta, diabetes tipo 2 y enfermedades cardiovasculares.

\section{Objetivo}

El objetivo del presente trabajo fue investigar los efectos de ocho semanas de ejercicio aeróbico regular y la estimulación eléctrica craneal (CES) sobre la hormona relacionada con el estrés, el factor neurotrófico, y los estados de ánimo en mujeres obesas.

\section{Método}

Los sujetos de este estudio fueron 36 mujeres coreanas de mediana edad con obesidad. Los sujetos fueron divididos aleatoriamente en un grupo control (CON, $n=12$ ), un grupo de ejercicio aeróbico (EX, $n=12$ ) y un grupo de ejercicio y CES aeróbico grupo (EX + CES, $n=12$ ). CES se llevó a cabo con una corriente estimulador de electroterapia craneal micro durante 20 minutos a $100 \mu \mathrm{A}$ y $0,5 \mathrm{~Hz}$. El ejercicio aeróbico consistía en carrera en cinta rodante durante 40 minutos a $70 \%$ de reserva de frecuencia cardíaca de los sujetos (HRR). El estado de ánimo se midió por el perfil de los estados de

Exercise Physiology Laboratory, Department of Physical Education, Yonsei University, Seoul, Republic of Korea.

Sports and Health Care Major, College of Humanities and Arts, Korea National University of Transportation, Chungju-si, Republic of Korea.

Department of Physical Education, College of Arts and Physical Education, Dong-A University, Busan, Republic of Korea.

Correspondence: Hee-Tae Roh, College of Arts and Physical Education, Department of Physical Education, Dong-A University, 37 Nakdong-daero 550 beon-gil, Hadan-dong, Saha-gu, Busan 604-714, Republic of Korea. Phone: 82-51-200-7835. Fax: 82-51-200-7805. E-mail: dau0409@dau.ac.kr

Received first version: December 16, 2015. Second version: May 27, 2016. Third version: August 19, 2016. Accepted: August 26, 2016. 
ánimo (POMS), y la extracción de sangre se realizó para examinar los niveles de las hormonas relacionadas con el estrés (cortisol y la hormona adrenocorticotrópica [ACTH]) y factores neurotróficos (factor neurotrófico derivado del cerebro [BDNF] y factor de crecimiento nervioso [NGF]) antes y después de cada intervención.

\section{Resultados}

Los niveles de cortisol en plasma, así como las puntuaciones de tensión-ansiedad, depresión, abatimiento, y fatiga-inercia, se redujeron significativamente después de la intervención, en comparación con las mediciones previas a la intervención en los grupos EX y EX + CES $(p<.05)$. Los niveles séricos de BDNF, los niveles de NGF en suero $y$ las puntuaciones Vigor-Actividad aumentaron significativamente des- pués de la intervención, en comparación con las mediciones previas a la intervención en los grupos EX y EX+CES $(p<.05)$. Sin embargo, no hubo diferencia significativa en estos hallazgos entre la EX y los grupos EX + CES ( $p>$.05).

\section{Discusión y conclusión}

Estos resultados sugieren que el entrenamiento con ejercicio aeróbico podría mejorar el estado de ánimo de las mujeres obesas de mediana edad por medio de una disminución en el cortisol sérico y un aumento en el suero BDNF y NGF.

Palabras clave: Ejercicio aeróbico, estimulación de electroterapia craneal, estado de ánimo, obesidad.

\section{INTRODUCTION}

Obesity, a global pandemic, has been reported as a potential cause of various diseases, including high blood pressure, type-2 diabetes, cardiovascular disease, cerebrovascular disease, and musculoskeletal disease. ${ }^{1}$ In addition, obesity is associated with the development of several mental disorders. $^{2}$

Among the mental disorders caused by obesity are depressive disorder and anxiety disorder. Obesity and depressive symptoms mutually affect each other in a bidirectional manner. Depressive symptoms frequently cause increased appetite and weight, ${ }^{3}$ while raising the risk of obesity by reducing physical activity. ${ }^{4}$ Prolonged depressive mood leads to overeating, increasing the risk of weight gain. ${ }^{5}$ Medicines for treating mood disorder and anxiety disorder can also cause weight gain..$^{6,7}$ Conversely, obesity can raise the risk of mental diseases, ${ }^{8}$ particularly in women who are vulnerable to depressive symptoms because of social prejudice against obese women. ${ }^{9}$ According to a large-scale epidemiology study, the prevalence of depression was four times higher among obese women than among overweight or normalweight women. ${ }^{10}$ In the case of middle-aged women, reduced estrogen secretion caused by menopause leads to increased body fat, raising the risk of obesity and, subsequently, mental disorders as depression and anxiety. ${ }^{11}$

Noninvasive brain stimulation is the method to achieve neuromodulation without surgical treatment through the safe local stimulation of a specific area of the brain using magnetism or electricity. Repetitive transcranial magnetic stimulation (rTMS), transcranial direct current stimulation (tDCS), and cranial electrotherapy stimulation (CES) are used clinically for the improvement of brain functioning and mental health. ${ }^{12,13}$ Of these, CES has been approved by US Food and Drug Administration (FDA) as a noninvasive, prescriptive medical treatment for depression, anxiety, and insomnia. ${ }^{14}$ Kirsch and Nichols (2013) suggested that CES treatment could be very effective for improving the symp- toms of depression, anxiety, and insomnia. ${ }^{13}$ However, it is not clear yet whether CES treatment can induce changes in the plasma indicators that reflect various mood states. Conflicting results have been reported in this regard. For example, Liss and Liss (1996) reported significant increases of tryptophan and endorphins as well as a significant decrease in serum cortisol after CES treatment in both males and females aged between 22 and $70 .{ }^{15}$ However, in contrast, Lee et al. (2013) reported that CES treatment for preoperative patients resulted in a significant reduction in preoperative anxiety but no significant changes in serum concentrations of stress hormones cortisol and adrenocorticotropic hormone (ACTH). ${ }^{16}$

In contrast, advanced studies have reported that obesity can cause psychiatric disorders, such as depression, anxiety disorder, schizophrenia, and bipolar disorder. The main factors are hypothalamus-pituitary-adrenal (HPA) axis disturbances, neurotransmitter imbalances, and neuroprogression, along with excessive fat accumulation. ${ }^{3}$ It has also been reported that a deficiency of brain-derived neurotrophic factor (BDNF), one of the main regulatory factors in neuronal development and synaptic function, is related to the increase in weight. ${ }^{17,18}$ Thus, the purpose of this study was to examine the effects of regular aerobic exercise and CES on stress hormone levels accompanying HPA axis activation, serum BDNF levels, and changes in mood state among middle-aged obese women.

\section{METHOD}

\section{Subjects}

A minimum sample size of 21 participants was deemed necessary for statistical significance, based on a repeated analysis of variance measures, with a $3 \times 2$ design, an anticipated statistical power of 0.80 , an alpha error probability of 0.05 , and an effect size of 0.40 (G-power program 3.1.3, Germany). The subjects of this study were 36 middle-aged obese 
women aged 50 to 59 years who visited the Physical Education Department at Yonsei University in Seoul, Republic of Korea from March 3 to May 30, 2014.

All subjects had to meet the following criteria before enrollment in the research: 1 . a BMI above $25 \mathrm{~kg} / \mathrm{m}^{2} ; 2$. no participation in regular physical activity programs; 3 . no chronic health problems or smoking; 4 . no history of cardiovascular, metabolic, or respiratory disease; and 5. at least 6 months since menopause.

All subjects voluntarily participated in this study and were sufficiently informed of the possible risk factors associated with the experiment. The subjects gave their consent, which included the right to withdraw from the study at any time. The study was conducted in accordance with the principles outlined in the Declaration of Helsinki. Randomization was performed using a simple randomization tool through an Internet site (Random.org) that assigns random numbers by the flip of a coin. The characteristics of the subjects are shown in Table 1.

\section{Anthropometric measurements}

Anthropometric measures, which were taken one week before the beginning of interventions, included the measurement of height, body composition, and maximal oxygen consumption $\left(\mathrm{VO}_{2} \mathrm{max}\right)$. Height and body composition were measured using a stadiometer (SECA213; SECA, Hamburg, Germany) and a bio-impedance analysis (BIA) device (Inbody720; Biospace, Seoul, Korea), respectively. $\mathrm{VO}_{2} \max$ was measured on a treadmill (Q65; Quinton, Seattle, WA, USA) according to the Bruce protocol based on the breathby-breath method with each subject wearing a gas analyzer (MetaMax 3B; Cortex, Leipzig, Germany). All anthropometric measures were repeated in the same fashion after 8 weeks of intervention to examine changes in the degree of obesity and cardiorespiratory fitness.

\section{Study design}

Subjects were randomly assigned to a 12-member control group (CON), an aerobic exercise group (EX), or an aerobic exercise and cranial electrotherapy stimulation (CES) group $(E X+C E S)$. Every group received eight weeks of intervention, except for the CON group whose members maintained their own lifestyles with no intervention.

The EX group was subjected to three sessions of 40-minute treadmill running a week; each session included a 10-minutes of warm-up stretching before the exercise and 10-minutes of cool-down stretching after the exercise, according to exercise prescription guidelines for obese subjects. ${ }^{19}$ Exercise intensity of treadmill running was set at $70 \%$ of heart rate reserve (HRR) determined using Karvonen's formula with the resting heart rate (HRrest) and maximum heart rate (HRmax) calculated during the $\mathrm{VO}_{2}$ max test. Heart rate was controlled within a $\pm 5 \%$ range of the target heart rate using a wireless heart rate analyzer (Polar a5; Polar, Finland). The EX group was given identical ear clip electrodes to the EX + CES group, but no electric current was given. The EX + CES group was subjected to the same treadmill running sessions as the EX group, in addition to CES treatment after each session. The CES treatment was administered three times a week using a micro current cranial electrotherapy stimulator (Alpha-Stim 100; Electromedical Products International, Mineral Wells, TX, USA). Based on the Alpha-Stim manual and the previous research of Lee et al. (2013), current and frequency were set at $100 \mu \mathrm{A}$ and $0.5 \mathrm{~Hz}$, respectively. Clip electrodes were attached to both earlobes to deliver 20 minutes of treatment. ${ }^{16}$

\section{Blood collection and analysis method}

Eight milliliters of blood were collected from the antecubital vein of each subject before and after each intervention

Table 1. Characteristics of the subjects

\begin{tabular}{|c|c|c|c|c|c|c|c|}
\hline \multirow[b]{2}{*}{ Variable } & \multicolumn{2}{|c|}{$\operatorname{CON}(n=12)$} & \multicolumn{2}{|c|}{$\operatorname{EX}(n=12)$} & \multicolumn{2}{|c|}{$\mathrm{EX}+\mathrm{CES}(n=12)$} & \multirow[b]{2}{*}{$\mathrm{p}$} \\
\hline & Before & After & Before & After & Before & After & \\
\hline Age (years) & \multicolumn{2}{|c|}{$54.67 \pm 3.03$} & \multicolumn{2}{|c|}{$54.83 \pm 2.79$} & \multicolumn{2}{|c|}{$54.75 \pm 2.45$} & 0.99 \\
\hline Height $(\mathrm{cm})$ & \multicolumn{2}{|c|}{$158.21 \pm 6.60$} & \multicolumn{2}{|c|}{$159.32 \pm 3.98$} & \multicolumn{2}{|c|}{$156.27 \pm 4.07$} & 0.34 \\
\hline Weight (kg) & $65.83 \pm 6.42$ & $66.43 \pm 6.90$ & $68.26 \pm 6.76$ & $61.87 \pm 5.07^{\#}$ & $65.77 \pm 8.28$ & $60.48 \pm 7.13^{\#}$ & 0.63 \\
\hline BMI $\left(\mathrm{kg} / \mathrm{m}^{2}\right)$ & $26.28 \pm 1.67$ & $26.51 \pm 1.82$ & $26.87 \pm 2.15$ & $24.38 \pm 1.81^{\#}$ & $26.90 \pm 2.82$ & $24.73 \pm 2.38 \#$ & 0.75 \\
\hline Fat mass $(\mathrm{kg})$ & $23.53 \pm 2.64$ & $23.70 \pm 3.03$ & $23.88 \pm 3.35$ & $19.51 \pm 3.26^{\#}$ & $25.10 \pm 5.49$ & $20.89 \pm 4.26^{\#}$ & 0.46 \\
\hline Body fat (\%) & $38.74 \pm 3.56$ & $38.66 \pm 3.71$ & $36.82 \pm 3.33$ & $33.15 \pm 4.67^{\#}$ & $37.92 \pm 4.29$ & $32.98 \pm 4.05^{\#}$ & 0.61 \\
\hline $\mathrm{VO}_{2} \max (\mathrm{ml} / \mathrm{kg} / \mathrm{min})$ & $27.89 \pm 3.74$ & $27.29 \pm 3.15$ & $27.73 \pm 3.29$ & $33.25 \pm 4.18$ & $27.63 \pm 2.94$ & $32.88 \pm 3.72^{5}$ & 0.98 \\
\hline
\end{tabular}

Data are presented as mean \pm standard deviation.

$\mathrm{CON}$, control group.

EX, aerobic exercise group.

EX + CES, aerobic exercise and cranial electrotherapy stimulation group.

$\mathrm{BMI}$, body mass index.

$\mathrm{VO}_{2}$ max, maximal oxygen consumption.

\#significantly lower than before within group $(p<.05)$.

significantly higher than before within group $(p<.05)$.

$p$ as determined using the one-way ANOVA for each of the three groups at baseline. 
using a 22-gauge needle, a serum separator tube (Becton Dickinson, Franklin Lakes, NJ, USA), and an ethylenediamine tetra-acetic acid tube (Becton Dickinson, Franklin Lakes, NJ, USA). Collected blood samples were centrifuged for 15 minutes at $3000 \mathrm{rpm}$ and then stored at $-80{ }^{\circ} \mathrm{C}$ until analysis.

\section{Plasma stress-related hormone analysis method}

The analyses of plasma cortisol were determined by the radioimmunoassay (RIA) using a commercially available Cortisol Coat-A-Count ${ }^{\circledR}$ kit (Cat. no. TKCO1; Siemens, Los Angeles, CA, USA). Similarly, ACTH concentrations were determined by the immunoradiometric assay (IRMA) using a commercially available ACTH IRMA (CT) kit (Cat. no. RE11081; IBL-International, Hamburg, Germany).

\section{Blood neurotrophic factor analysis method}

The analyses of serum brain-derived neurotrophic factor (BDNF) were carried out using a human BDNF ELISA Kit (Cat. no. DBD00; R\&D Systems, Minneapolis, MN, USA), while nerve growth factor (NGF) analyses used an NGF sandwich ELISA Kit (Cat. no. CYT304; Chemicon, Temecula, CA, USA). A microplate reader (EMax; Molecular device, Sunnyvale, CA, USA) was used to measure absorbance at $450 \mathrm{~nm}$ for quantification.

\section{Measurement of mood state}

Change in mood state was measured using the Korean edition of the profile of mood states (K-POMS); K-POMS is an adaptation by Kim et al. (2003) of the profile of mood states (POMS) developed by McNair et al. (1992) whose reliability and validity has been verified..$^{20,21}$ Based on a five-point Likert scale, this questionnaire was composed of a total of 65 questions dealing with six subcategories: Tension-Anxiety, Depression-Dejection, Anger-Hostility, Vigor-Activity, Fatigue-Inertia, and Confusion-Bewilderment.

\section{Statistical analysis}

Statistical analyses were performed with the SPSS version 21.0 for Windows (SPSS Inc., Chicago, IL, USA). Data are presented as the mean \pm standard deviation (SD), unless otherwise stated. A three group (CON, EX, and EX + CES) by time point (before and after intervention) repeated measures ANOVA was used to examine the effect of CES and exercise training on plasma cortisol and ACTH, serum BDNF and NGF, and POMS response. When significant group $X$ time interactions occurred, simple main effects were assessed using one-way ANOVA and paired $t$-tests. Levels of significance were set at 0.05 .

\section{RESULTS}

\section{Changes in plasma stress-related hormone levels}

The plasma cortisol and ACTH levels for the three groups before and after intervention are shown in Table 2. Following intervention, repeated ANOVA measures demonstrated a significant difference across group by time interaction for plasma cortisol $(F[2,33]=12.33, p<.01)$ levels. Plasma cortisol levels were significantly decreased after intervention when compared with those before intervention in the EX $(t=3.98)$ and EX + CES $(t=4.86)$ groups $(p<.05)$. In addition, plasma cortisol levels after intervention were significantly lower in EX and EX + CES groups than in CON group $(F[2,33]=4.44, p<.05)$. In contrast, plasma ACTH levels were not significantly different among groups and time points $(F[2,33]=0.26, p=.77)$.

\section{Changes in serum neurotrophic factor levels}

The serum BDNF and NGF levels for the three groups before and after intervention are shown in Table 2. Following intervention, repeated ANOVA measures demonstrated a significant difference across groups by time interaction for serum BDNF $(F[2,33]=6.60, p<.01)$ and $\operatorname{NGF}(F[2,33]$ $=5.90, p=.01$ ) levels. Serum BDNF and NGF levels were significantly increased after intervention when compared with those before intervention in $\operatorname{EX}(t=-3.84$ and $t=-4.42$, respectively) and EX + CES $(t=-4.20$ and $t=-4.17$, respectively) groups $(p<.05)$. In addition, serum BDNF levels after intervention were significantly higher in the EX and EX + CES groups than in the CON group $(F[2,33]=4.52, p<.05)$.

\section{Changes in POMS scores}

The POMS scores for the three groups before and after intervention are shown in Table 2. Following intervention, repeated ANOVA measures demonstrated a significant difference across groups by time interaction for Tension-Anxiety $(F[2,33]$ $=5.90, p=.01)$, Depression-Dejection $(F[2,33]=3.58, p=.04)$, Vigor-Activity $(F[2,33]=13.26, p<.01)$, and Fatigue-Inertia $(F[2,33]=3.81, p=.03)$ scores. Vigor-Activity scores were significantly increased, while Tension-Anxiety, Depression-Dejection, and Fatigue-Inertia scores were significantly decreased after intervention when compared with those before intervention in the $\operatorname{EX}(t=-6.63, t=6.66, t=5.82$, and $t=6.43$, respectively) and EX + CES $(t=-4.57, t=3.08, t=5.46$, and $t=4.14$, respectively) groups $(p<.05)$. In addition, Vigor-Activity scores after intervention were significantly higher in EX and EX + CES groups than in CON group $(F[2,33]=4.49, p<.05)$. In contrast, Anger-Hostility and Confusion-Bewilderment scores were not significantly different among all groups and time points $(F[2$, $33]=.02, p=.98$, and $F[2,33]=.04, p=.96$, respectively). 


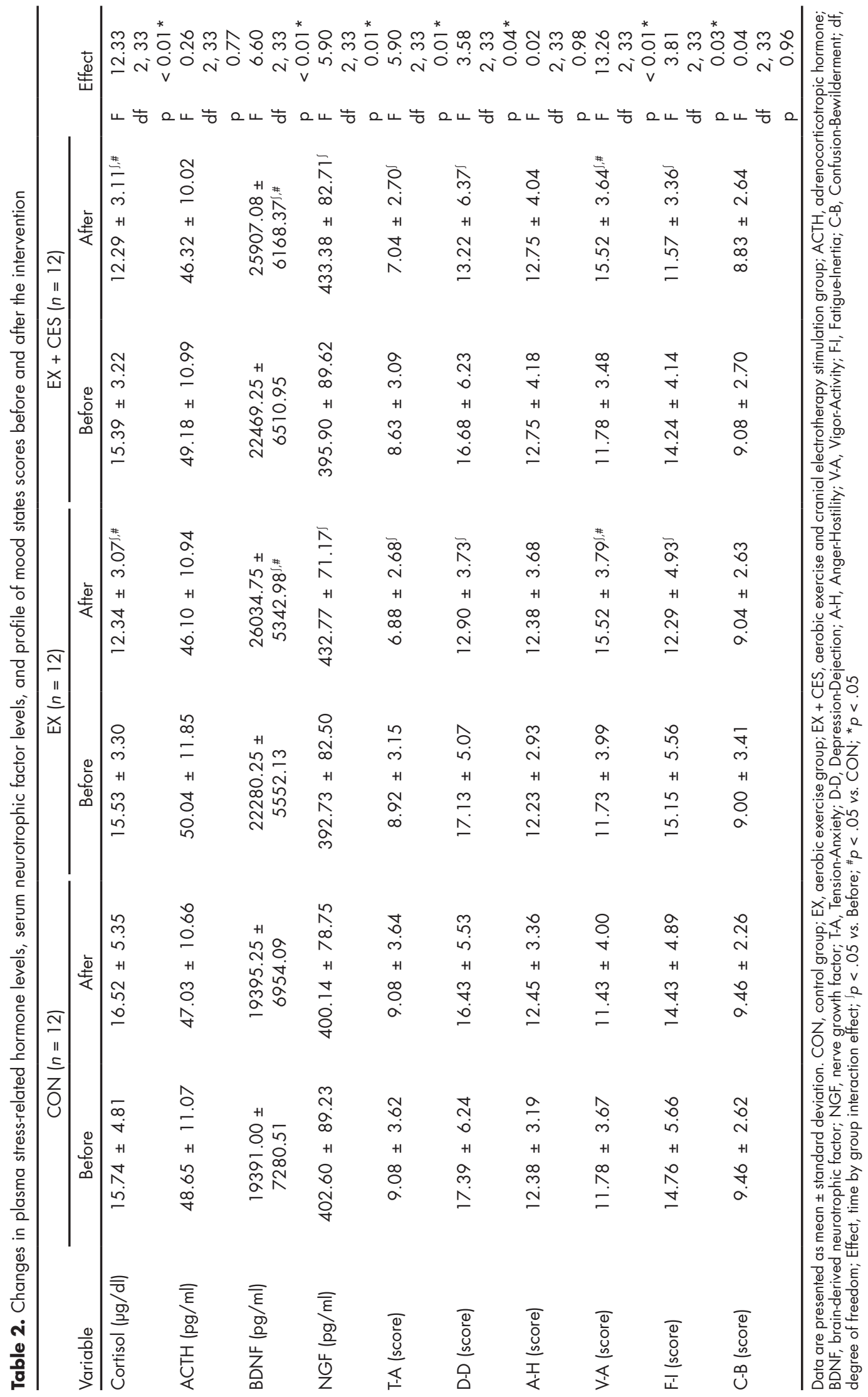




\section{DISCUSSION AND CONCLUSION}

Hypothalamic-pituitary-adrenal (HPA) axis hyperactivation is a characteristic stress response in mammals ${ }^{22}$ reflected by high plasma cortisol and ACTH levels. ${ }^{23}$ HPA axis abnormalities are the most common neuroendocrinological disorders found in patients with depression, ${ }^{24}$ and with other mood disorders. ${ }^{25}$ It has been reported that $30-50 \%$ of the patients with major depressive disorder have evidence of HPA axis dysfunction. ${ }^{24,26}$ Excessive cortisol secretion in patients with depression was first reported by Board et al. (1956), ${ }^{27}$ while there have also been reports of significantly higher levels of ACTH secretion in such patients when compared to those in a healthy population. ${ }^{28}$ The present research analyzed plasma cortisol and ACTH levels to investigate changes in stress-related hormones in obese middle-aged women according to the combined intervention of aerobic exercise and CES. The results show that there was a significant decrease in the plasma cortisol levels after intervention in the EX and EX + CES groups; these decreases were statistically significant when compared to those in the CON group. Such a result is likely due to the alleviation of the HPA axis response by exercise training. This interpretation is supported by the suggestion of Wittert et al. (1996) that although the HPA axis hyperactivation by stress leads to elevated secretion of cortisol, repeated exposure to a particular stress as endurance training could make the HPA axis less responsive to the initial stress. ${ }^{29}$ Similarly, Fry et al. (1991) reported that exercise training could decrease plasma stress hormone concentrations by gradually improving the stability of the pituitary system. ${ }^{30}$ Unlike plasma cortisol levels, plasma ACTH levels in the present research did not show significant change following intervention. This result agrees with the findings of Traustadóttir et al. (2005) who investigated the effects of aging and fitness level on HPA axis response in women in their 20's and 60 's. After exposing them to stress using matt stress reactivity protocol (MSRP), ${ }^{31}$ he observed no significant difference in ACTH level according to fitness level, although cortisol concentrations were significantly lower in patients with higher than average fitness levels. ${ }^{31}$ This research led to a similar suggestion that exercise training could alleviate HPA axis response to psychological stress. Similarly, in the present study, based on the significant increase of $\mathrm{VO}_{2} \mathrm{max}$ after treatment in the EX and EX + CES groups, we believe that the main cause of the changes in plasma cortisol and ACTH levels is the increase in cardiorespiratory fitness due to exercise training.

Neurotrophic factors, such as BDNF and NGF, play crucial roles in regulating the proliferation, migration, survival, and synaptic plasticity of neurons. ${ }^{32}$ BDNF and NGF are not only involved in the regulation of the neuroendocrine system but can also pass through the bloodbrain barrier into the systemic circulation. For this reason, peripheral BDNF and NGF levels have been suggested as biomarkers for diagnosis and disease monitoring in mood disorders such as depression. ${ }^{33,34}$ For example, based on the meta-analyses of 11 studies, Sen et al. (2008) showed significantly lower BDNF levels in patients with depression than in the healthy control group; this finding suggests that serum BDNF levels could serve as a biomarker for diagnosing psychiatric disorders. ${ }^{33}$ Similarly, Wiener et al. (2015) reported significantly lower serum NGF levels in patients with major depressive disorder than in a healthy control group. ${ }^{34}$ The present research analyzed serum BDNF and NGF levels in order to assess changes in neurotrophic factors in obese middle-aged women following aerobic exercise or a combination of aerobic exercise and CES. There were significant increases of serum BDNF and NGF levels after intervention in the EX and EX + CES groups; particularly, serum BDNF levels after intervention were significantly higher in these groups compared to those in the CON group. This result is in agreement with previous studies that concluded that regular aerobic exercise training caused a significant increase of serum BDNF level in obese subjects. ${ }^{35,36}$ Our findings also support the result of a previous study that found that dietary changes and aerobic exercise training induced significantly higher levels of BDNF and NGF expression in the hippocampus of obese rats. ${ }^{37}$ In addition, a previous report indicated that dysfunction of BDNF and NGF, as well as of their respective receptors tropomyosin receptor kinase $B(\operatorname{TrkB})$ and tropomyosin receptor kinase A (TrkA), could contribute to the pathogenesis of both depression and obesity. ${ }^{38}$ Accordingly, the significant increases of serum BDNF and NGF levels in the present research are potentially due to the improvement of obesity by aerobic exercise training. Indeed, after treatment, the EX and EX + CES groups showed significant reductions in body weight, BMI, fat mass, and body fat. This finding is supported by the work of Schulte-Herbrüggen et al. (2009) who reported a negative correlation $(r=-0.69, p<.0001)$ between body weight and serum BDNF levels. ${ }^{38}$ Similarly, Danaalamdari et al. (2014) reported a negative correlation $(r=-0.43, p=.032)$ between BMI and serum NGF levels. ${ }^{39}$

Obesity is one of the factors that threatens mental health by causing such abnormal conditions as depression, anxiety, and panic disorders; positive relationships between obesity and such psychiatric disorders have been mainly observed in women. ${ }^{40}$ The present research utilized the profile of mood states (POMS) to verify the effect of aerobic exercise and the combined intervention of aerobic exercise and CES on the mood state of obese middle-aged women. Following intervention, the EX and EX + CES groups showed significant decreases in Tension-Anxiety, Depression-Dejection, and Fatigue-Inertia scores, as well as a significant increase in the Vigor-Activity score. This result supports prior studies that utilized POMS to demonstrate significant improvement of mood state following aerobic 
exercise. ${ }^{41-43}$ Specifically, Sakuragi and Sugiyama (2006) reported that four weeks of walking exercise resulted in a significant decrease in the scores in the Tension-Anxiety and Depression-Dejection subscales of POMS. ${ }^{41}$ According to the report of Petajan et al. (1996), 10 weeks of aerobic training resulted in a significant increase of the Fatigue-Inertia score. ${ }^{42}$ In addition, Brown et al. (1992) reported that participation in a 9-week aerobic exercise program could bring about significant increase of the Vigor-Activity score. ${ }^{43}$ The changes in POMS scores in the present research also suggest the positive impact of aerobic exercise training in improving the mood state, and the major contributors to this improvement are thought to be the decrease in cortisol and increases of BDNF and NGF. Such an interpretation is supported by previous reports which point out that regular exercise could be effective for the maintenance and improvement of mental health by reducing distress and negative affect while promoting positive benefits for the cognitive, emotional, and motor domains. ${ }^{44,45}$ These reports have also suggested that such beneficial effects of exercise could be explained by the maintenance of HPA axis homeostasis, as well as by the elevation of the expression levels of neurotrophic factors such as BDNF and NGF. ${ }^{44,46}$

On the other hand, there was no significant difference in any variable between the EX and EX + CES groups. Therefore, it is judged that CES treatment did not induce changes in stress-related hormone response, neurotrophic factor activation, and mood state. There have been conflicting results as to whether CES treatment can alleviate and treat such mental diseases as depression and anxiety and induce changes in related biomarkers. For example, Barclay and Barclay (2014) reported that CES treatment was effective for alleviating the symptoms of anxiety and depression, ${ }^{47}$ but the systematic review of Kavirajan et al. (2014) revealed no positive effect of CES treatment on acute depression. ${ }^{48}$ In addition, Liss and Liss (1996) suggested that an $18 \%$ decrease in plasma cortisol levels after CES treatment was due to the reduced stress response, ${ }^{15}$ but Lee et al. (2013) reported that CES treatment showed an alleviating effect on preoperative anxiety involving no change in plasma cortisol and ACTH levels. ${ }^{16}$ There are also conflicting results from a limited amount of research concerning the effect of such noninvasive brain stimulation as repetitive transcranial magnetic stimulation (rTMS) and transcranial direct current stimulation (tDCS) on neurotrophic factor expression. Zanardini et al. (2006) reported significant increases of serum BDNF after rTMS treatment in drug resistant depressed patients, ${ }^{49}$ but Brunoni et al. (2014) reported no significant change in BDNF after tDCS treatment. ${ }^{50}$ Thus, while noninvasive brain stimulation, including CES, can directly regulate the plasticity of the cranial nerves and provide some alleviation and treatment of the symptoms of mental diseases, such as depression and anxiety, patients' responses to noninvasive brain stimulation could differ ac- cording to their individual characteristics (such as the severity of disease, age and gender). There is also a recent report indicating that the efficacies of noninvasive brain stimulation could differ according to the genetic characteristics of patients. ${ }^{51}$ Moreover, effective timing for applying noninvasive brain stimulation, as well as proper cranial stimulation parameters, still need to be clearly verified..$^{52}$ Accordingly, follow-up studies would need to verify these parameters using various research techniques (such as factorial, randomized, sham-controlled trial) in consideration of the patients' diverse characteristics (such as age, gender, and the type and severity of the disease).

In conclusion, our overall results suggest that aerobic exercise training can exert positive effects on the mood state of obese middle-aged women through a decrease in the cortisol levels and increases in BDNF and NGF. However, CES treatment did not have a significant positive effect on mental health status in middle-aged obese women, as the results of CES treatment combined with exercise were not significantly different from those of treatment with exercise alone.

Nevertheless, the present research has some limitations. This was a pilot study conducted at a single organization and therefore had a small number of subjects. In addition, it was previously reported that improvements in mental health status using noninvasive brain stimulation treatment, including CES, can vary according to age, sex, and the type and degree of disease. ${ }^{51}$ Thus, future studies should recruit a greater number of subjects in order to comprehensively investigate the effects of CES treatment.

\section{Funding}

This study was supported by research funds from Dong-A University.

\section{Conflict of interest}

The authors declare that there is no conflict of interest.

\section{REFERENCES}

1. Villablanca PA, Alegria JR, Mookadam F, Holmes DR Jr et al. Nonexercise activity thermogenesis in obesity management. Mayo Clin Proc 2015;90(4):509-519.

2. Lopresti AL, Drummond PD. Obesity and psychiatric disorders: commonalities in dysregulated biological pathways and their implications for treatment. Prog Neuropsychopharmacol Biol Psychiatry 2013;45:92-99.

3. Carter FA, Bulik CM, Joyce PR. Direction of weight change in depression. J Affect Disord 1994;30(1):57-60.

4. Friedman KE, Reichmann SK, Costanzo PR, Musante GJ. Body image partially mediates the relationship between obesity and psychological distress. Obes Res 2002;10(1):33-41.

5. Hasler G, Pine DS, Gamma A, Milos G et al. The associations between psychopathology and being overweight: a 20-year prospective study. Psychol Med 2004;34(6):1047-1057.

6. Rapaport MH, Thase ME. Translating the evidence on atypical depression into clinical practice. J Clin Psychiatry 2007;68(4):e11.

7. Schwartz TL, Nihalani N, Jindal S, Virk S et al. Psychiatric medication induced obesity: a review. Obes Rev 2004;5(3):115-121. 
8. Kivimäki M, Batty GD, Singh-Manoux A, Nabi H et al. Association between common mental disorder and obesity over the adult life course. Br J Psychiatry 2009;195(2):149-155.

9. Puhl RM, Brownell KD. Psychosocial origins of obesity stigma: toward changing a powerful and pervasive bias. Obes Rev 2003;4(4):213-227.

10. Keddie AM. Associations between severe obesity and depression: results from the National Health and Nutrition Examination Survey, 2005-2006. Prev Chronic Dis 2011;8(3):A57.

11. Stachowiak G, Pertyński T, Pertyńska-Marczewska M. Metabolic disorders in menopause. Prz Menopauzalny 2015;14(1):59-64.

12. Fregni F, Pascual-Leone A. Technology insight: noninvasive brain stimulation in neurology-perspectives on the therapeutic potential of rTMS and tDCS. Nat Clin Pract Neurol 2007;3(7):383-393.

13. Kirsch DL, Nichols F. Cranial electrotherapy stimulation for treatment of anxiety, depression, and insomnia. Psychiatr Clin North Am 2013;36(1):169-176.

14. Kirsch DL, Price LR, Nichols F, Marksberry JA et al. Military service member and veteran self reports of efficacy of cranial electrotherapy stimulation for anxiety, posttraumatic stress disorder, insomnia, and depression. US Army Med Dep J 2014;46-54.

15. Liss S, Liss B. Physiological and therapeutic effects of high frequency electrical pulses. Integr Physiol Behav Sci 1996;31(2):88-95.

16. Lee SH, Kim WY, Lee CH, Min TJ et al. Effects of cranial electrotherapy stimulation on preoperative anxiety, pain and endocrine response. J Int Med Res 2013;41(6):1788-1795.

17. Noble EE, Billington CJ, Kotz CM, Wang C. The lighter side of BDNF. Am J Physiol Regul Integr Comp Physiol 2011;300(5):1053-1069.

18. Schwartz E, Mobbs CV. Hypothalamic BDNF and obesity: found in translation. Nat Med 2012;18(4):496-497.

19. ACSM. ACSM's guidelines for exercise testing and prescription. Philadelphia: Lippincott williams \& wilkins; 2006.

20. Kim EJ, Lee SI, Jeong DU, Shin MS et al. Standardization and reliability and validity of the Korean edition of Profile of Mood States (K-POMS). Sleep Med Psychophysiol 2003;10(1):39-51.

21. McNair DM, Lorr M, Droppleman LF. Profile of mood states manual. San Diego: Educational and Industrial Testing Service; 1992.

22. Rodriguez-Herreros B, de Grave DD, Lopez-Moliner J, Brenner E et al. Shifted visual feedback of the hand affects reachability judgments in interception. Vision Res 2013;88:30-37.

23. Schulz A, Vögele C. Interoception and stress. Front Psychol 2015;6:993.

24. Tichomirowa M, Keck M, Schneider H, Paez-Pereda M et al. Endocrine disturbances in depression. J Endocrinol Invest 2005;28(1):89-99.

25. Watson S. Mackin P. HPA axis function in mood disorders. Psychiatry 2006;5(5):166-170.

26. Scott LV, Dinan TG. Vasopressin as a target for antidepressant development: an assessment of the available evidence. J Affect Disord 2002;72(2):113-124.

27. Board F, Persky H, Hamburg DA. Psychological stress and endocrine functions; blood levels of adrenocortical and thyroid hormones in acutely disturbed patients. Psychosom Med 1956;18(4):324-333.

28. Deuschle M, Schweiger U, Weber B, Gotthardt U et al. Diurnal activity and pulsatility of the hypothalamus-pituitary-adrenal system in male depressed patients and healthy controls. J Clin Endocrinol Metab 1997;82(1):234-238.

29. Wittert GA, Livesey JH, Espiner EA, Donald RA. Adaptation of the hypothalamopituitary adrenal axis to chronic exercise stress in humans. Med Sci Sports Exerc 1996;28(8):1015-1019.

30. Fry RW, Morton AR, Keast D. Overtraining in athletes. An update. Sports Med 1991;12(1):32-65.

31. Traustadóttir T, Bosch PR, Matt KS. The HPA axis response to stress in women: effects of aging and fitness. Psychoneuroendocrinology. 2005;30(4):392-402.

32. Bartkowska K, Turlejski K, Djavadian RL. Neurotrophins and their receptors in early development of the mammalian nervous system. Acta Neurobiol Exp (Wars) 2010;70(4):454-467.
33. Sen S, Duman R, Sanacora G. Serum brain-derived neurotrophic factor, depression, and antidepressant medications: meta-analyses and implications. Biol Psychiatry 2008;264(6):527-532.

34. Wiener CD, de Mello Ferreira S, Pedrotti Moreira F, Bittencourt G et al. Serum levels of nerve growth factor (NGF) in patients with major depression disorder and suicide risk. J Affect Disord 2015;184:245248.

35. Araya AV, Orellana X, Godoy D, Soto L et al. Effect of exercise on circulating levels of brain-derived neurotrophic factor (BDNF) in overweight and obese subjects. Horm Metab Res 2013;45(7):541-544.

36. Lee SS, Yoo JH, Kang S, Woo JH et al. The effects of 12 weeks regular aerobic exercise on brain-derived neurotrophic factor and inflammatory factors in juvenile obesity and type 2 diabetes mellitus. J Phys Ther Sci 2014;26(8):1199-1204.

37. Woo J, Shin KO, Park SY, Jang KS et al. Effects of exercise and diet change on cognition function and synaptic plasticity in high fat diet induced obese rats. Lipids Health Dis 2013;12:144.

38. Schulte-Herbrüggen $\mathrm{O}$, Fuchs E, Abumaria N, Ziegler A et al. Effects of escitalopram on the regulation of brain-derived neurotrophic factor and nerve growth factor protein levels in a rat model of chronic stress. J Neurosci Res 2009;87(11):2551-2560.

39. Danaalamdari L, Kheirouri S, Alizadeh M, Aslani, Z. The association between serum NGF and BDNF levels with obesity in patients with major depression. Nutrition Food Sciences Research 2014;1(1):96.

40. Lykouras L, Michopoulos J. Anxiety disorders and obesity. Psychiatriki 2011;22(4):307-313.

41. Sakuragi S, Sugiyama Y. Effects of daily walking on subjective symptoms, mood and autonomic nervous function. J Physiol Anthropol 2006;25(4):281-289.

42. Petajan JH, Gappmaier E, White AT, Spencer MK et al. Impact of aerobic training on fitness and quality of life in multiple sclerosis. Ann Neurol 1996;39(4):432-441.

43. Brown SW, Welsh MC, Labbé EE, Vitulli WF et al. Aerobic exercise in the psychological treatment of adolescents. Percept Mot Skills 1992;74(2):555-560.

44. Archer T, Josefsson T, Lindwall M. Effects of physical exercise on depressive symptoms and biomarkers in depression. CNS Neurol Disord Drug Targets 2014;13(10):1640-1653.

45. Ströhle A. Physical activity, exercise, depression and anxiety disorders. J Neural Transm (Vienna) 2009;116(6):777-784.

46. Duman RS. Neurotrophic factors and regulation of mood: role of exercise, diet and metabolism. Neurobiol Aging 2005;1:88-93.

47. Barclay TH, Barclay RD. A clinical trial of cranial electrotherapy stimulation for anxiety and comorbid depression. J Affect Disord 2014;164:171-177.

48. Kavirajan HC, Lueck K, Chuang K. Alternating current cranial electrotherapy stimulation (CES) for depression. Cochrane Database Syst Rev 2014;7:CD010521.

49. Zanardini R, Gazzoli A, Ventriglia M, Perez J et al. Effect of repetitive transcranial magnetic stimulation on serum brain derived neurotrophic factor in drug resistant depressed patients. J Affect Disord 2006;91(1):83-86.

50. Brunoni AR, Machado-Vieira R, Zarate CA Jr, Vieira EL et al. BDNF plasma levels after antidepressant treatment with sertraline and transcranial direct current stimulation: results from a factorial, randomized, sham-controlled trial. Eur Neuropsychopharmacol 2014;24(7):1144-1151.

51. Cheeran B, Talelli P, Mori F, Koch G et al. A common polymorphism in the brain-derived neurotrophic factor gene (BDNF) modulates human cortical plasticity and the response to rTMS. J Physiol 2008;586 (Pt 23):5717-5725.

52. Ridding MC, Rothwell JC. Is there a future for therapeutic use of transcranial magnetic stimulation? Nat Rev Neurosci 2007;8(7):559-567. 